\title{
Archéopages
}

Archéopages Archéologie et société

$45 \mid 2017$

De la terre au pot

\section{Un fœtus en bocal inhumé au XIX siècle}

\section{Anne Richier}

\section{OpenEdition}

Journals

Édition électronique

URL : https://journals.openedition.org/archeopages/3587

DOI : 10.4000/archeopages.3587

ISSN : 2269-9872

Éditeur

INRAP - Institut national de recherches archéologiques préventives

\section{Édition imprimée}

Date de publication : 1 mars 2018

Pagination : $142-143$

ISSN : 1622-8545

\section{Référence électronique}

Anne Richier, « Un fœetus en bocal inhumé au xı ${ }^{e}$ siècle », Archéopages [En ligne], 45 | 2017, mis en ligne le 01 janvier 2020, consulté le 04 juin 2021. URL : http://journals.openedition.org/archeopages/ 3587 ; DOI : https://doi.org/10.4000/archeopages.3587 
Période

Époque contemporaine

\section{Inhumation d'un foetus en bocal}

Anne Richier

Inrap, responsable d'opération, UMR 7268 «ADES »
Site

Quartier des Crottes

Marseille

Bouches-du-Rhône

Date

Février-août 2013 et janvier-mai 2014

Équipe

Anthropologie

Isabelle Bouchez

Inrap

Clémence Delteil

Assistance publique -

Hôpitauxde Marseille

Mélissa Niel

Facultéde médecine de Marseille,

UMR 7268, "ADES»

Étude du petit mobilier

Édith Coustaud

Inrap

Denis Michel

Inrap [ill. 1]. Un avant-bras en connexion à l'intérieur du réceptacle de bois attestait l'inhumation d'un sujet adulte, de sexe indéterminé. Placé entre le cercueil et le bord de la fosse, un bocal circulaire en verre, fermé par un couvercle, contenait des restes osseux [ill. 2]. Ils correspondent à un foetus complet très bien conservé, d'un âge compris entre 4 et 5 mois lunaires (la détermination de l'âge au décès a été effectuée à partir de mesures prises sur les os longs et les clavicules, Adalian, 2001 ; Sherer et al., 2006). Son caractère complet indique qu'il s'agit d'un dépôt primaire, c'est-à-dire que c'est un petit cadavre qui a été déposé à l'intérieur du bocal [ill. 3]. Le bouchon en verre ne devait pas être hermétique et de l'eau a remplacé le liquide initialement versé (sans doute de l'alcool, le formol n'étant utilisé à des fins conservatoires qu'à la fin du Xıx ${ }^{\mathrm{e}}$ siècle), ce qui a entraîné la totale décomposition des chairs. L'examen minutieux des ossements a mis en évidence une anomalie développementale du crâne qui a sans doute occasionné le décès prématuré du fotus. Il s'agit d'une agénésie de l'os pariétal gauche et d'un développement inégal des os frontaux, à mettre en relation avec plusieurs maladies rares d'origine congénitale, génétique ou secondaire. Il peut aussi, avec réserves, s'agir d'une hydrocéphalie (diagnostic de Clémence Delteil à partir de l'étude de Mélissa Niel). En revanche, il reste difficile de savoir si cette anomalie était apparente, ouvrant la piste d'une conservation à but tératologique (science des «monstruosités »). Cette découverte singulière a suscité plusieurs questions : pour quelles raisons ce fœetus conservé dans un bocal a-t-il été placé dans un cimetière? Comment expliquer le geste des fossoyeurs plaçant un tel « objet» contre un cercueil lors de l'inhumation?

$\mathrm{Au} \mathrm{XIX}{ }^{\mathrm{e}}$ siècle, les foetus en bocal faisaient partie du quotidien : il était possible d'en voir dans les musées d'anatomie, les officines de pharmacie, les foires, voire chez les particuliers. L'on sait par exemple que la mère de Paul Verlaine a précautionneusement conservé ses fœtus issus de fausses couches dans des bocaux (Buisine, 1995). De nombreux écrivains ou poètes du XIX ${ }^{\mathrm{e}}$ siècle, comme Émile Zola ou Maurice Mac-Nab, les ont évoqués dans leurs œuvres.

Aujourd'hui encore, nombre de facultés de médecine ou musées de tératologie conservent des collections de foetus en bocal. Les raisons de cette conservation et exposition sont directement liées à l'évolution des sciences et de la médecine; dès le $\mathrm{XVI}^{\mathrm{e}}$ siècle, les « avortons » suscitaient l'intérêt des médecins et des intellectuels, mais plutôt comme support à des interrogations philosophiques sur les débuts de la vie.

Dès lors, comment expliquer incomplète puisque largement détruite par une fosse de spoliation
1. Vue partielle de la sépulture comportant des restes de cercueil de bois et un bocal, déposé au fond de la fosse au moment de l'inhumation du sujet adulte.

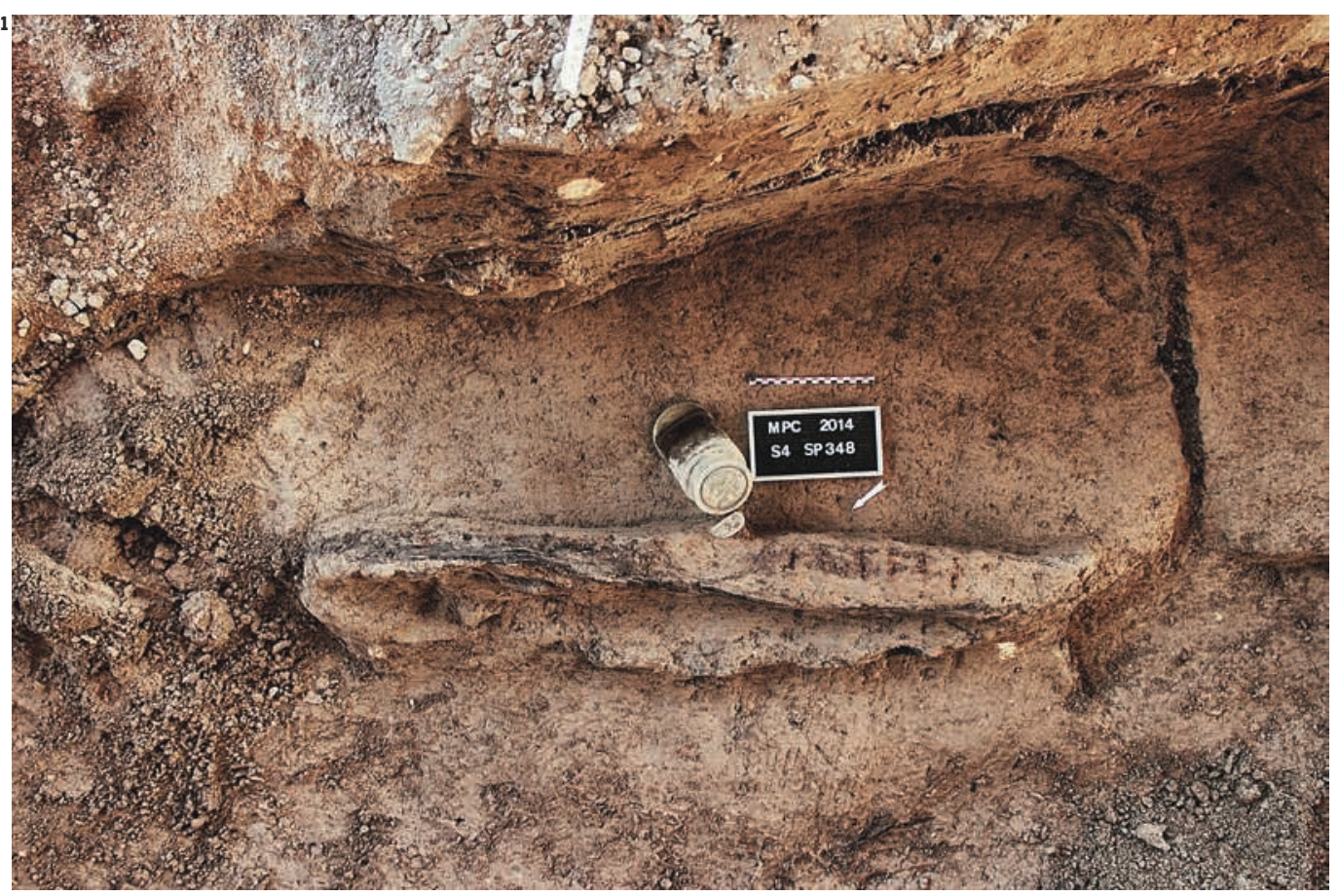


2. Le bocal en verre, de $155 \mathrm{~mm}$ de haut et de $96 \mathrm{~mm}$ de diamètre, à moitié rempli d'eau et comportant des restes osseux à sa base. II s'agit d'un pot d'apothicaire de forme très courante dans les productions de verre pharmaceutique du xix siècle.

3. L'étude des vestiges contenus dans le bocal a montré qu'il s'agissait d'un fœetus entier, âgé de 4 à 5 mois in utero.
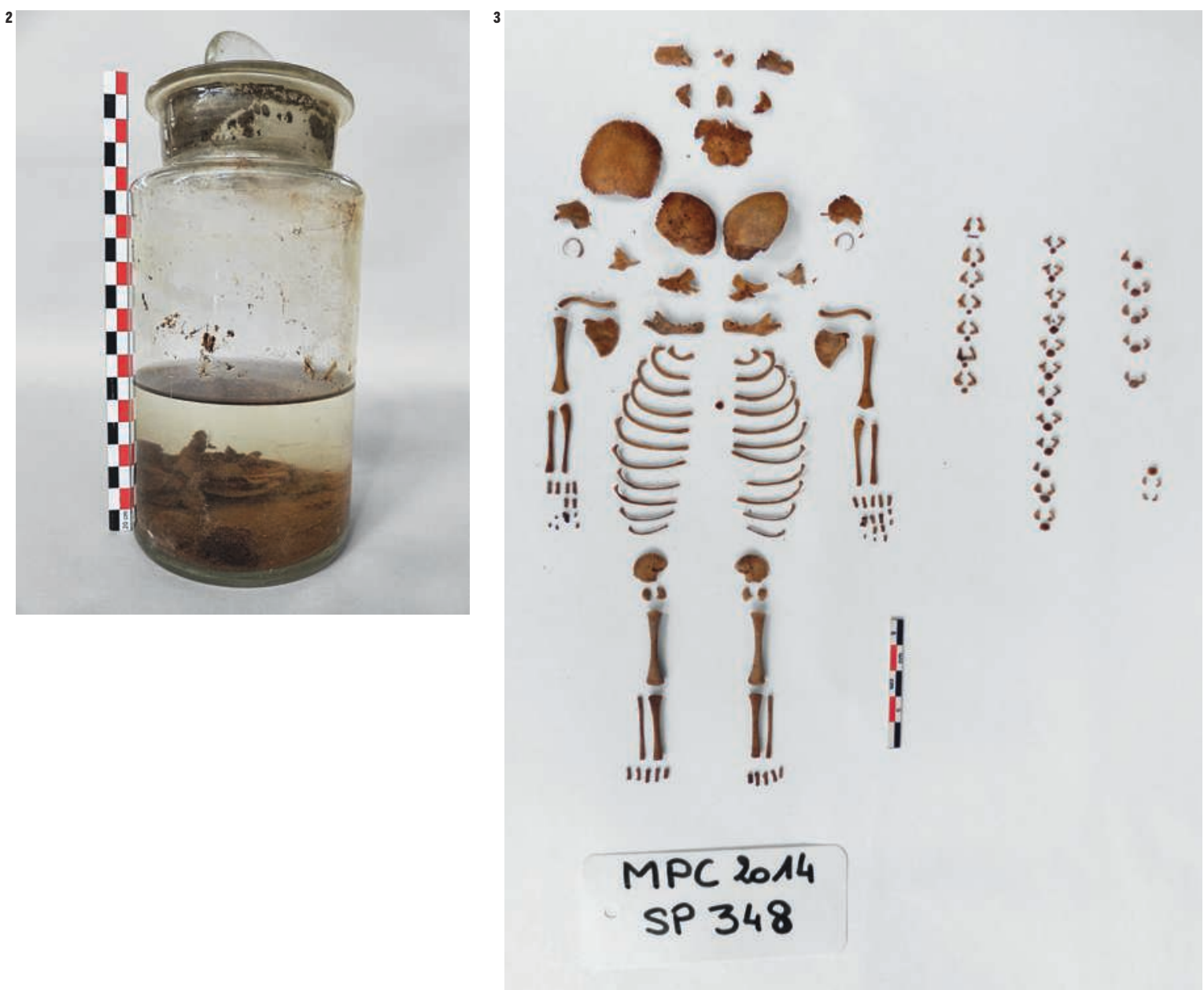

le cimetière de Marseille ? Les pistes pour tenter de comprendre ce dépôt particulier sont minces. Il peut être le reflet d'une volonté familiale comme d'un geste opportuniste face à un « objet encombrant ». Un dépouillement intégral des actes de sépultures dans les registres de catholicité de la paroisse des Crottes a été effectué, mais n'a livré aucune information remarquable, hormis quelques mentions de pharmaciens dans les métiers.

$\mathrm{Au}$-delà des raisons qui ont conduit à déposer ce foetus en bocal dans une sépulture, cette découverte pose la question du statut des toutpetits morts avant terme et non viables. Car si le principe même d'enfermer un corps dans un bocal signe sa réification («chosification»), son dépôt dans un cimetière indique au contraire un geste funéraire, donc une attitude d'être humain envers l'un de ses semblables. Les « avortons » étaient-ils considérés comme des êtres humains au
$\mathrm{XIX}^{\mathrm{e}}$ siècle ? Le sont-ils aujourd'hui ? Un foetus non viable (d'un âge inférieur à 22 semaines d'aménorrhée ou cinquième mois de grossesse et/ou un poids inférieur à $500 \mathrm{~g}$ selon l'Organisation mondiale de la Santé) est nécessairement issu d'un avortement spontané (fausse couche) ou déclenché. L'abondante littérature judiciaire qui traite des procès de «faiseuses d'anges » reste assez silencieuse sur le sort réservé aux fœetus expulsés de force : il y est au mieux question d'enterrement clandestin dans le jardin, mais, dans la majorité des cas, ils sont jetés dans la rue, le puits, la fosse à purin, voire brûlés (Morel, 2003). Les fotus n'étaient donc pas considérés par le peuple comme des êtres humains mais comme des résidus, des déchets dont il fallait se débarrasser au plus vite, d'autant plus que la législation en cours criminalisait l'avortemen dès les débuts de la grossesse (Code pénal de 1810). Depuis les progrès de la médecine au XVIII ${ }^{\mathrm{e}}$ siècle en effet, scientifiques et théologiens s'étaient accordés pour donner au fœtus un statut d'être humain dès sa conception. Aujourd'hui, les foetus n'ont un statut juridique que lorsqu'ils sont nés viables et vivants (aptes à vivre et présentant des signes de vie) ou à partir du septième mois de grossesse pour les mort-nés. Quant aux fœtus en bocal, ils sont des objets muséographiques, leur statut ne posait donc pas problème au XIX ${ }^{e}$ siècle, contrairement à aujourd'hui, même si la législation comporte encore de grandes lacunes sur le sujet (Gasnier, 2013).

La découverte du bocal inhumé dans le cimetière de Marseille pose plus de question qu'elle n'amène de réponses. Il est toutefois possible d'attester, à travers son dépôt dans l'espace communautaire des morts, qu'une part d'humanité a été rendue à un petit être non viable, d'abord considéré comme objet de curiosité.

\section{Références bibliographiques}

Adalian P., 2001, Évaluation multiparamétrique de la croissance foetale - Applications à la détermination de l'âge et du sexe, Marseille, Université de la Méditerranée/Aix-Marseille II Faculté de Médecine de Marseille, $269 \mathrm{p}$.

Buisine A., 1995, Verlaine. Histoire d'un corps, Paris, Tallandier, $536 \mathrm{p}$.

GASNIER J.-P., 2013, « L'enfant mort-né, du futur au plus-que-parfait», Techniques \& Culture, 6o, p. 30-43

Morel M.-F., 2003, « Histoire de l'avortement ( $2^{\mathrm{e}}$ partie) : Le vécu des avortements aux XIX ${ }^{e}$ et $\mathrm{XX}^{\mathrm{e}}$ siècles ", Réalités en gynécologie obstétrique, 77, p. 77-83.

Sherer D. et al., 2006, « Fetal clavicle length throughout gestation: a nomogram ", Ultrasound in Obstetrics and Gynecology, 27(3), p. 306-310. 